\title{
Structural and thermal behavior of compact core-shell nanoparticles: Core instabilities and dynamic contributions to surface thermal stability
}

\author{
Andrés Aguado and José M. López \\ Departamento de Física Teórica, Universidad de Valladolid, Valladolid 47071, Spain \\ (Received 7 July 2005; revised manuscript received 13 September 2005; published 14 November 2005)
}

\begin{abstract}
An orbital-free density-functional-theory molecular dynamics technique is applied to investigate the minimum-energy structure and meltinglike transition of $\mathrm{Cs}_{55}, \mathrm{Li}_{13} \mathrm{Na}_{32} \mathrm{Cs}_{42}$, and $\mathrm{Li}_{55} \mathrm{Cs}_{42}$ nanoparticles. Icosahedral packing is found to be optimal for homogeneous $\mathrm{Cs}_{55}$, as expected. Heterogeneous particles show a complete segregation of Cs atoms to the cluster surface, and form perfect core-shell structures, that is, structures where each atomic species occupies and completes a different concentric atomic shell. For $\mathrm{Li}_{13} \mathrm{Na}_{32} \mathrm{Cs}_{42}$, the size mismatch between atomic species forming different shells leads to polyicosahedral packing. For $\mathrm{Li}_{55} \mathrm{Cs}_{42}$, however, the size mismatch is huge and perfect polyicosahedral ordering is frustrated, resulting in more complex structural behavior. The three clusters investigated share the same surface shell, formed by 42 Cs atoms, and comparison of their melting behaviors helps to rationalize the increased thermal stability of the cluster surface upon alloying. $\mathrm{Cs}_{55}$ melts homogeneously at approximately $85 \mathrm{~K}$. Both $\mathrm{Li}_{13} \mathrm{Na}_{32} \mathrm{Cs}_{42}$ and $\mathrm{Li}_{55} \mathrm{Cs}_{42}$ show a substantial thermal stability, compared to $\mathrm{Cs}_{55}$ and other alloy compositions where a perfect core-shell structure does not appear. We demonstrate that an important contribution to this increased thermal stability in the nanoalloys comes from the large difference in the atomic masses of the constituent particles, which results in a poor coupling of atomic vibrations along the radial direction. We also give arguments to show that the meltinglike transition in these clusters is triggered by the thermal instability of interior rather than surface atoms. Segregation of Cs atoms to the cluster surface is fully maintained in the liquid state, so that core and surface shells form two inmiscible liquid layers.
\end{abstract}

DOI: $10.1103 /$ PhysRevB.72.205420

PACS number(s): 61.46.+w, 36.40.Ei, 36.40.Sx, 31.15.Qg

\section{INTRODUCTION}

Physical and chemical properties of heterogeneous metallic clusters (or nanoalloys) depend not only on cluster size but also on composition. Compared to homogeneous metallic nanoparticles, this provides additional flexibility that can be employed to tailor materials with specific properties adequate to each particular application, for example, in heterogeneous catalysis. ${ }^{1}$ The number of experimental ${ }^{2-8}$ and

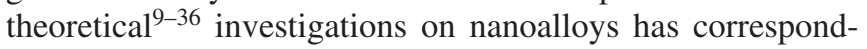
ingly increased in the last decade.

The most basic property of a nanocluster is its structure. Knowledge of the minimum energy atomic configuration is a prerequisite for the correct determination of many other (thermal, electronic, etc.) cluster properties. While the major structural features of most homogeneous metal nanoparticles (icosahedral, decahedral, face-centred-cubic atomic packings, tendency to amorphization, etc.) are reasonably well understood, the corresponding situation is much less advanced for heterogeneous clusters. To start with, there is the additional complication of compositional ordering, which leads to the concept of "homotop" introduced by Jellinek and Krissinel. ${ }^{10}$ Homotops are isomers sharing the same inherent topological structure but differing in the allocation of different species in the atomic sites. The lowest energy structure of a heterogeneous cluster is determined by a competition between several effects, such as the relative bond strengths of the atomic species involved, their size and surface tension mismatches, or the minimization of the strain energy associated with departures from the optimal interatomic distances. All together, these factors determine the segregation/mixing preferences as well as the specific geometry of the cluster.

Most of the investigations mentioned above have focused on nanoalloys formed by transition metal elements, as these are the most promising for industrial applications in catalysis or optical devices. However, much general knowledge on the structural and thermal behaviors of nanoalloys may also be obtained from the study of different clusters, such as those formed by inert gases interacting through a Lennard-Jones (LJ) potential ${ }^{28,29}$ or simple alkali metals. ${ }^{30-36}$ Due to their "simplicity," both LJ and Na clusters have been employed in the past to gather a lot of information about the physical properties of homogeneous clusters. In particular, conjoint experimental $^{37-39}$ and theoretical ${ }^{40-51}$ efforts have made possible that the meltinglike transition in unsupported sodium clusters begins to be understood. An important part of the information obtained on these simple systems may be later transferred to the understanding of similar properties of more complex systems such as transition metal clusters. The situation will most probably be similar with heterogeneous clusters, which provides motivation for exploring the properties of LJ and alkali nanoalloys.

For example, Rossi et al. ${ }^{21}$ and Rapallo et al. ${ }^{24}$ have recently shown that alloying of some transition or noble metals stabilizes core-shell polyicosahedral structures such as the compact anti-Mackay icosahedron for $\mathrm{Ni}_{13} \mathrm{Ag}_{32}$. The same preference for polyicosahedral and/or polytetrahedral packing has been independently obtained by Calvo and Yurtsever $^{28}$ and Doye and Meyer ${ }^{29}$ for mixed LJ clusters and by Aguado and López for binary and ternary mixtures of alkali metals. ${ }^{35,36}$ Anti-Mackay overlayers are not specially stable for homogeneous clusters because of the associated 
bond strain, but they are very stable in those mixtures which present (a) a sufficiently large size mismatch and (b) a lower surface tension of the atomic species with larger radius. In this case, both core $(\mathrm{Ni} / \mathrm{Na} / \mathrm{Ar})$ and surface $(\mathrm{Ag} / \mathrm{Cs} / \mathrm{Xe})$ atoms may have interatomic distances close to optimal in the polyicosahedral structure, which explains its stabilization. For metallic nanoalloys, the correlation between coordination number and optimal bond length (which is a consequence of the many-body character of metallic interactions) also favors polyicosahedral packing in those cases. When the size mismatch and/or surface tension difference is small, Rossi et al. $^{25}$ and Aguado and López ${ }^{35}$ have shown that polyicosahedral ordering is not so favored, and partial mixing of the atomic species may be energetically preferred over the formation of a perfect core-shell nanoparticle.

Regarding the thermal properties, nanoalloys may also present more complex behavior than homogeneous clusters. Calvo and Yurtsever ${ }^{28}$ have shown that solid-solid transformations between homotops may occur at low temperatures as precursors to the meltinglike transition, if those homotops have similar binding energies. Aguado and Lópe ${ }^{35}$ have found that surface premelting effects in $\mathrm{Na}-\mathrm{Cs}$ nanoalloys are more important than in homegenous alkali clusters, for those compositions not corresponding to a perfect core-shell structure (that is, when the surface shell, formed by Cs atoms, is not of monatomic thickness). The same preponderance of premelting effects was found in Li-Na nanoalloys due to incomplete surface segregation of $\mathrm{Na}$ atoms, mostly at finite temperatures. Premelting was significantly suppressed in $\mathrm{Li}_{13} \mathrm{Na}_{30} \mathrm{Cs}_{12}$, where each atomic species occupies a different radial shell of monatomic thickness, even if the surface layer formed by Cs atoms is not complete. ${ }^{36}$ Finally, Rossi et al. ${ }^{21}$ reported that the melting point of $\mathrm{Ag}_{27} \mathrm{Ni}_{7}$, a perfectly compact core-shell nanoparticle, was higher than those of homogeneous Ag or Ni clusters of a similar size.

In this paper, we report the results of extensive orbitalfree $a b$ initio molecular dynamics (OFAIMD) simulations of the meltinglike transition in $\mathrm{Cs}_{55}, \mathrm{Li}_{13} \mathrm{Na}_{32} \mathrm{Cs}_{42}$, and $\mathrm{Li}_{55} \mathrm{Cs}_{42}$. The main goal of this work is to analyze the enhanced thermal stability of two compact core-shell alkali nanoalloys which share the same surface shell (formed by a complete overlayer of $42 \mathrm{Cs}$ atoms) and compare it to the thermal stability of $\mathrm{Cs}_{55}$, a homogeneous cluster also with the same surface shell. We will show that the two perfect core-shell nanoalloys melt at a considerably higher temperature than $\mathrm{Cs}_{55}$ or other nanoalloys of compositions not corresponding to a perfect core-shell structure. In particular, the surface shell formed by $\mathrm{Cs}$ atoms remains solid up to temperatures higher than the evaporation threshold temperature of $\mathrm{Cs}_{55}$. The most significant results are that (1) we demonstrate that a very important contribution to this thermal stabilization comes from the large mass difference between several atomic species, and not just to the larger strength of $\mathrm{Na}-\mathrm{Cs}$ and Li-Na bonds as compared to Cs-Cs bonds; (2) we demonstrate that the meltinglike transition in these clusters is triggered by thermal instability of the core rather than the surface shell. A secondary goal is to compare in detail the melting behavior of $\mathrm{Cs}_{55}$ with that previously reported by Aguado $^{43}$ for the same cluster employing a less accurate realization of the OFAIMD technique. Our present results show that $\mathrm{Cs}_{55}$ undergoes homogeneous melting without a separate surface melting stage, similarly to the situation found in $\mathrm{Na}$ clusters. ${ }^{50,51}$

The rest of the paper is structured as follows: Section II presents a brief summary of our theoretical method, a full account of which can be found in our recent report on the OFAIMD technique. ${ }^{34}$ Section III describes our results on the structural and thermal properties of $\mathrm{Cs}_{55}, \mathrm{Li}_{13} \mathrm{Na}_{32} \mathrm{Cs}_{42}$, and $\mathrm{Li}_{55} \mathrm{Cs}_{42}$. Finally, Sec. IV offers some concluding remarks.

\section{THEORY}

For a given spatial configuration of atoms, we evaluate the energy of the cluster and the force acting on each atom by employing density functional theory (DFT) in its Hohenberg-Kohn $(\mathrm{HK})^{53}$ representation, where the valence electron density stands as the basic variable, thus avoiding employment of auxiliary one-particle orbitals as in its KohnSham $(\mathrm{KS})^{54}$ representation. The details of our implementation of this so-called orbital-free DFT scheme have been described in previous work, ${ }^{33,34,40-43}$ so we just present briefly the main technical issues. The electronic kinetic energy functional of the electron density is approximated by a generalization of the gradient expansion around the homogeneous limit through second order, ${ }^{53,55-57} T[n]=T_{T F}[n]+\lambda T_{v W}[n]$, usually denoted $T F \lambda v W$ functional. This means that we keep the local Thomas-Fermi term and the lowest order density gradient correction, and that the numerical factor multiplying the gradient term $(\lambda)$ may be considered a fitting parameter. The local density approximation (LDA) is used for exchange and correlation. ${ }^{58,59}$ The ionic field acting on the electrons is represented by the local pseudopotentials of Fiolhais et al. ${ }^{60}$ The parameters defining these pseudopotentials and $\lambda$ are obtained from a force-matching procedure, as explained in our recent work on $\mathrm{Na}$ clusters. ${ }^{50}$ Briefly, we perform KSLDA calculations on a representative set of cluster structures (including several ordered isomers and also disordered ones obtained from preliminary high-temperature simulations) employing the SIESTA code, ${ }^{61}$ and find the parameters which best reproduce, within the OFAIMD technique, the atomic forces and energy differences between isomers given by the SIESTA code. The parameters resulting from this matching procedure are $\lambda=0.44$ and (employing the same notation as Fiolhais et $\left.a l .{ }^{60}\right) \quad \alpha(\mathrm{Li} / \mathrm{Na} / \mathrm{Cs})$ $=4.113 / 3.499 / 3.138, \quad R(\mathrm{Li} / \mathrm{Na} / \mathrm{Cs})=0.345 / 0.454 / 0.875$. The obtained pseudopotentials are much more accurate for clusters than those originally reported by Fiolhais et al. ${ }^{60}$ which were intended to reproduce a few bulk properties under linear response theory calculations. It is interesting to note that we obtain a $\lambda$ value very close to 0.4 . This value has very recently been found to be optimal also for bulk metal systems by Carter and co-workers. ${ }^{62}$ In fact, this functional reproduces KSLDA results with approximately the same accuracy as the fully nonlocal functional we employed in our recent work on Na clusters, ${ }^{50}$ and is simpler to use. The nonlocal functional is more transferable, however, when considering several clusters in a broad size range because it includes an explicit dependence on the cluster size.

The clusters under study are placed in a unit cell of a cubic superlattice with edge $38 \AA\left(\mathrm{Cs}_{55}\right.$ and $\left.\mathrm{Li}_{55} \mathrm{Cs}_{42}\right)$ and 
$41 \AA\left(\mathrm{Li}_{13} \mathrm{Na}_{32} \mathrm{Cs}_{42}\right)$, and the set of plane waves periodic in those superlattices, up to an energy cutoff of $20 \mathrm{Ryd}$, is used as a basis set to expand the valence electron density. Following Car and Parrinello, ${ }^{63}$ the coefficients of that expansion are regarded as generalized coordinates of a set of fictitious classical particles, and the corresponding Lagrange equations of motion for the electron density distribution are solved in order to determine the optimal electron density for each atomic configuration, as described in Ref. 34. Forces on atoms are then evaluated by using Hellmann-Feynman's theorem. Thus the dynamics of ions is not Car-Parrinello, but Born-Oppenheimer. The equations of motion are integrated using the Verlet algorithm, ${ }^{64}$ with a time step of 9 fs for $\mathrm{Cs}_{55}$ and 3 fs for both nanoalloys. We also conducted simulations for $\mathrm{Li}_{13} \mathrm{Na}_{32} \mathrm{Cs}_{42}$ and $\mathrm{Li}_{55} \mathrm{Cs}_{42}$ in which the masses of all particles were set equal to the Cs atomic mass (see next section). For these simulations, a time step of $7 \mathrm{fs}$ was employed. Several isokinetic MD runs were performed in order to obtain the caloric curve for each cluster. The total simulated time was approximately $13 \mathrm{~ns}$ for $\mathrm{Cs}_{55}$ and $10 \mathrm{~ns}$ for both heterogeneous clusters.

A number of different indicators was employed in order to analyze the meltinglike transition. First, the average internal temperature $T$ is defined from the equipartition theorem for the average kinetic energy:

$$
T=\frac{2\left\langle E_{k}\right\rangle}{(3 N-7) k_{B}}
$$

where we have taken into account the fact that the position of the center of mass of the cluster was fixed, the total angular momentum was held to zero, and the total kinetic energy of the cluster was kept constant during the simulations, thus reducing the number of degrees of freedom from $3 N$ to $3 N$ -7 . Note that the isokinetic distribution ${ }^{65-68}$ is the product of a canonical distribution of configurational degrees of freedom times a microcanonical distribution of kinetic degrees of freedom. The resulting velocity distribution is therefore not expected to be Maxwell-Boltzmann-like as the fluctuations in the kinetic energy term are suppressed. We nevertheless employ the kinetic temperature defined in Eq. (1) as a convenient operational parameter measuring the average kinetic energy of the cluster, both in equilibrium and nonequilibrium MD simulations. Although the average temperature value thus obtained is not very sensitive to the statistical sampling employed, fluctuations in finite size systems may be highly dependent on the statistical ensemble. The results shown in this paper apply to the isokinetic ensemble.

The rest of the melting indicators are (a) the root-meansquared bond-length fluctuation

$$
\delta=\frac{2}{N(N-1)} \sum_{i<j} \frac{\sqrt{\left\langle R_{i j}^{2}\right\rangle_{t}-\left\langle R_{i j}\right\rangle_{t}^{2}}}{\left\langle R_{i j}\right\rangle_{t}},
$$

where $R_{i j}$ is the distance between atoms $i$ and $j$; (b) shorttime averages of the "atomic equivalence indexes" 69

$$
\sigma_{i}(t)=\sum_{j}\left|\vec{R}_{i}(t)-\vec{R}_{j}(t)\right|
$$

(c) the averaged atomic distribution function, defined by
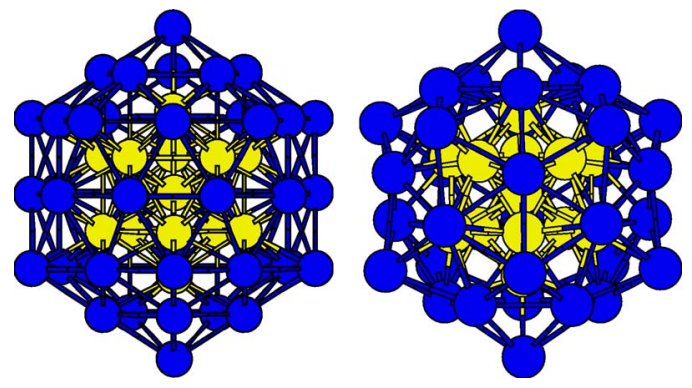

FIG. 1. (Color online) Comparison of Mackay (left) and antiMackay (right) growing on top of a 13-atom icosahedron. Surface and core atoms are given different shades of gray (colors) to help visualization. The 55-atom Mackay icosahedron is the ground state structure for $\mathrm{Cs}_{55}$. Anti-Mackay growing leads to the 45-atom polyicosahedral shell closing, which is favored for binary metallic systems with a large size mismatch and surface tension difference between the two components.

$$
d N_{a t}(r)=g(r) d r,
$$

where $d N_{a t}(r)$ is the number of atoms at distances from the center of mass between $r$ and $r+d r$; and (d) the temporal Fourier transform, $P(\omega)=2 \int_{0}^{\infty} C(t) \cos \omega t d t$, of the velocity autocorrelation function $C(t)=\left\langle\Sigma_{i} \vec{v}_{i}(t) \cdot \vec{v}_{i}(0)\right\rangle /\left\langle\sum_{i} v_{i}(0)^{2}\right\rangle$. $P(\omega)$ is the so-called power spectrum, proportional to the density of normal vibrational modes in the harmonic approximation.

\section{RESULTS}

\section{A. $\mathrm{Cs}_{55}$}

We have not employed an unbiased global optimization method to locate the ground state structure of $\mathrm{Cs}_{55}$. We have just locally optimized icosahedral, decahedral, and cuboctahedral isomers, together with some fragments of fcc (incomplete truncated octahedra) and bcc crystalline lattices, and obtained that the 2-shell Mackay icosahedron (see Fig. 1) has the lowest energy, which is the expected result for an alkali cluster of this size. This isomer remains stable upon heating up to the melting point, which gives further support to it as the most probable ground state isomer.

Figure 2 shows the caloric curve, as well as the temperature evolution of the rms bond-length fluctuation. Both indicators agree in locating the melting temperature at $T_{m}$ $\approx 85 \mathrm{~K}$. In the temperature interval $T=85-100 \mathrm{~K}$, it is very difficult to obtain fully converged $\delta$ values, which will most probably be larger than those shown in Fig. 2. Both Fig. 2 and a visual inspection of the MD trajectories show that $\mathrm{Cs}_{55}$ melts homogeneously. This is at variance with our previous findings for the same cluster, ${ }^{43}$ where a surface premelting stage was clearly identified at $90 \mathrm{~K}$, with homogeneous melting occuring at $130 \mathrm{~K}$. The main prediction from this work is therefore that the surface shell of $\mathrm{Cs}_{55}$ is a nonmelting surface.

In our previous work, ${ }^{43}$ we employed essentially the same OFAIMD technique but with a different energy functional. Specifically, the pseudopotential parameters as given by Fiolhais ${ }^{60}$ and the $T F \frac{1}{9} v W$ functional were employed. Figure 


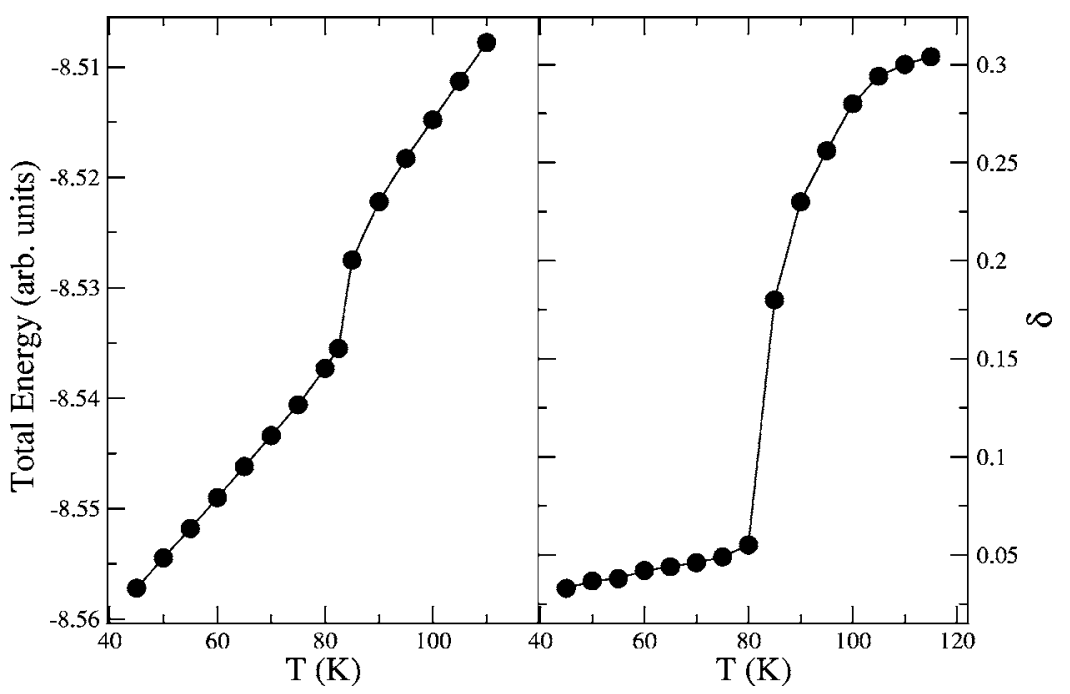

FIG. 2. Caloric curve (left) and rms bondlength-fluctuation parameter (right) of $\mathrm{Cs}_{55}$ as a function of the cluster internal temperature.

3 compares the atomic forces predicted by present and older OFAIMD realizations to KSAIMD SIESTA results. We have chosen the optimized icosahedral structure (zero atomic forces) predicted by the previous OFAIMD realization as an example. It is evident that the dynamics generated in Ref. 43 suffered from important quantitative errors as compared to the dynamics generated in the present study. Equilibrium distances calculated with present OFAIMD results agree to better than $1.0 \%$ with SIESTA equilibrium distances. The same is true for the relative stabilities of the several isomers considered (see above).

A high surface stability might be the reason for the absence of a separate surface melting stage prior to homogeneous melting in $\mathrm{Cs}_{55}$. To test this idea, we would like to devise a method to decide whether the melting is due to thermal instability of core or surface atoms. We have chosen to perform several nonequilibrium MD simulations, in which the velocities of the 13 core and 42 surface atoms are separately scaled to generate a thermal gradient along the radial direction. In this way we can associate, via Eq. (1), different local temperatures with the core and surface shells, as a measure of the inhomogeneous distribution of kinetic energies in those two regions. The results of these calculations, performed for several thermal gradients, are summarized in
Table I, which shows $T_{\text {core }}$ and $T_{\text {surface }}$ values for the highest temperature runs in which the cluster is still fully solidlike, together with the corresponding melting temperature values [although the definition of temperature away from equilibrium is always problematic, we employ here $T_{m}$ only as an operational measure of the average kinetic energy of the whole cluster, according to Eq. (1)]. The first important point is that the value of $T_{m}$ may be significantly affected by a thermal gradient along the radial direction: when the core shell is at a higher temperature than the surface shell, the melting point is lowered, and vice versa. The second important point is that all systems melt when $T_{\text {core }}$ approaches $85 \mathrm{~K}$, the equilibrium melting point value, irrespective of the value of $T_{\text {surface, }}$ which may be as low as $62 \mathrm{~K}$ or as high as $101 \mathrm{~K}$ just before melting. This provides, we believe, strong indication that it is the thermal instability of the core shell at approximately $85 \mathrm{~K}$ which ultimately drives the transition to the liquid phase for $\mathrm{Cs}_{55}$. As long as the thermal gradient is not huge, homogeneous melting results. We have run short simulations which demonstrate that much larger thermal gradients are needed to lead to a separate surface or core melting stage, but have not investigated this problem in detail.

By rescaling atom velocities by different factors in core and surface shells, we introduce an abrupt thermal interface

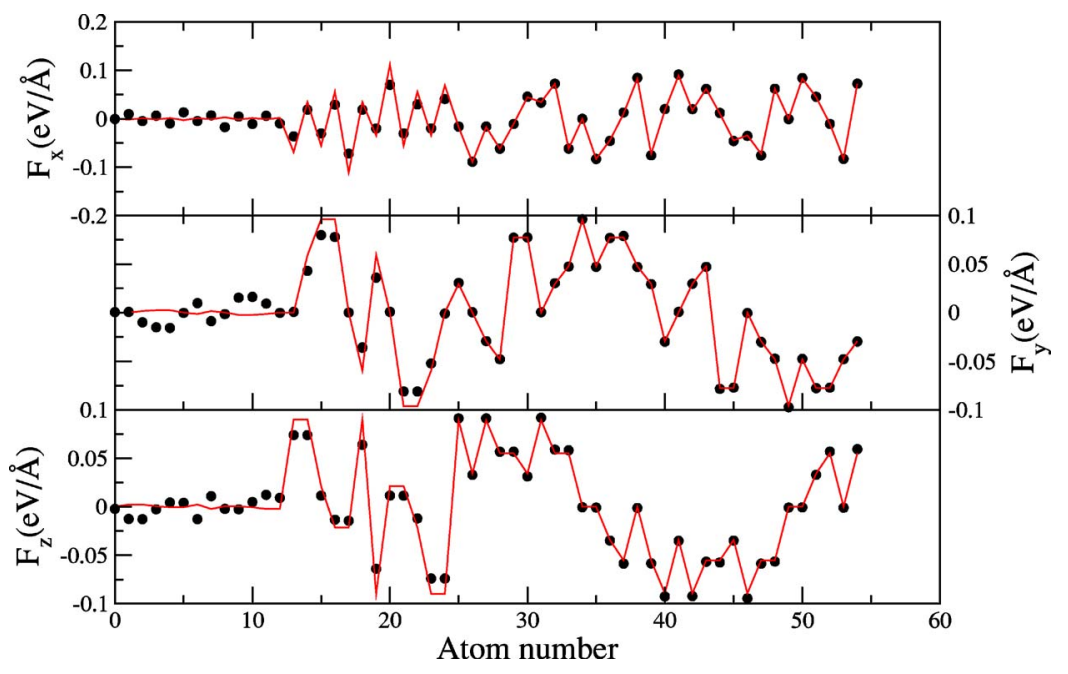

FIG. 3. (Color online) Comparison between SIESTA (full symbols) and present (continuous line) OFAIMD results for the interatomic forces of the $\mathrm{Cs}_{55}$ icosahedral atomic configuration which is optimal (zero atomic forces) for the previous OFAIMD realization (Ref. 43). Both SIESTA and present OFAIMD results produce interatomic forces significantly deviating from zero. 
TABLE I. Core and surface shell local temperatures (in K) for the highest temperature run in which the cluster remained fully solid, when several thermal gradients are imposed along the radial direction. The last column gives the melting temperature $T_{m}$ for each of these nonequilibrium runs.

\begin{tabular}{cccc}
\hline \hline Cluster & $T_{\text {core }}$ & $T_{\text {surface }}$ & $T_{m}$ \\
\hline $\mathrm{Cs}_{55}$ & 82 & 67 & 73 \\
& 81 & 62 & 69 \\
& 83 & 92 & 93 \\
& 82 & 101 & 100 \\
$\mathrm{Li}_{13} \mathrm{Na}_{32} \mathrm{Cs}_{42}$ & 155 & 127 & 145 \\
$\mathrm{Li}_{55} \mathrm{Cs}_{42}$ & 156 & 192 & 180 \\
& 166 & 132 & 155 \\
& 167 & 210 & 188 \\
\hline \hline
\end{tabular}

with an associated excess energy. To what extent the melting transition is affected by the presence of this interface is not a simple issue, and in any case is beyond the scope of this paper. We just want to call attention to the point that, no matter how abrupt this interface is, the cluster always melts when the average kinetic energy of interior atoms approaches that corresponding to the homogeneous melting point, which in our opinion is a significant conclusion.

\section{B. $\mathrm{Li}_{13} \mathrm{Na}_{32} \mathrm{Cs}_{42}$}

To find the ground state isomer of a heteroatomic cluster of this size is an extremely difficult task. Rapallo et al. ${ }^{24}$ and Rossi et al. ${ }^{25}$ have recently made a very important effort to locate putative global minima of some transition metal binary clusters with a genetic algorithm. However, when the potential energy landscape is sampled by DFT means, the number of energy evaluations required by global optimization methods such as genetic algorithms is prohibitive. In this work, we have performed three different simulated annealing runs starting from a liquid cluster and cooling at a rate of $0.1 \mathrm{~K} / \mathrm{ps}$. We have also relied upon the experience gained in our previous works on nanoalloys ${ }^{35,36}$ and constructed high-symmetry isomers by growing on top of $\mathrm{Li}_{13}$ icosahedral, decahedral, and cuboctahedral seeds, which are between the expected topological structures for metal clusters. For each of these isomers, we additionally performed a mild annealing simulation by heating the cluster to approximately $120 \mathrm{~K}$ (in any case, a temperature lower than the melting point) and cooling it down at a rate of $0.4 \mathrm{~K} / \mathrm{ps}$. In many cases, this has the effect of locating an isomer of the same symmetry as the original one but with a slightly lower energy.

The three simulated annealing runs resulted in complete surface segregation of Cs atoms, and two of them also resulted in an inner core shell formed by $13 \mathrm{Li}$ atoms, while in the third one some mixing of $\mathrm{Li}$ and $\mathrm{Na}$ atoms at the two core shells was observed, but this isomer had a higher energy. The structures obtained from the first two simulated annealing runs are almost identical to the one obtained by polyicosahedral growing on a $\mathrm{Li}_{13}$ icosahedron, shown in
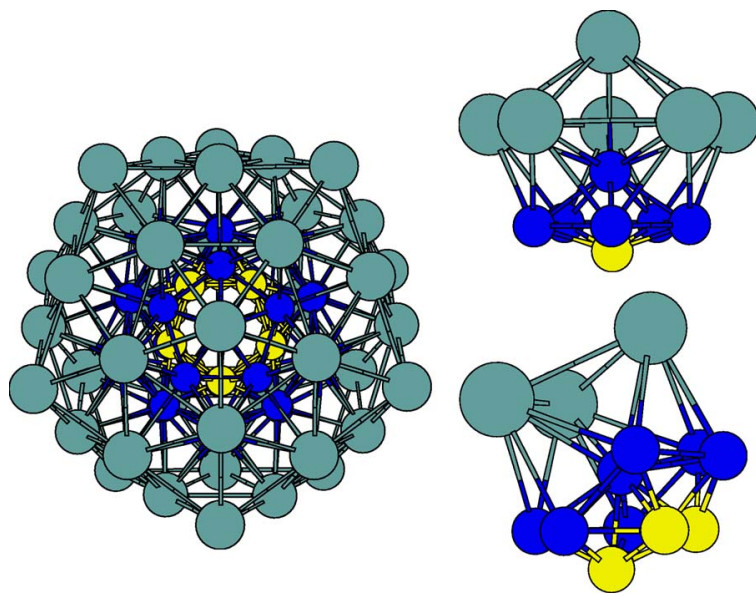

FIG. 4. (Color online) Lowest energy structure of $\mathrm{Li}_{13} \mathrm{Na}_{32} \mathrm{Cs}_{42}$ (left side). The core shell $\left(\mathrm{Li}_{13} \mathrm{Na}_{32}\right)$ is a perfect anti-Mackay icosahedron as shown in Fig. 1. On the right side, we show the different local coordination environments of the $\mathrm{Na}$ atoms occupying vertex (upper plot) and edge (lower plot) positions of $\mathrm{Li}_{13} \mathrm{Na}_{32}$. All $\mathrm{Na}$ atoms are placed at the center of a (slightly distorted) 13-atom icosahedron, which shows that the ground state structure of $\mathrm{Li}_{13} \mathrm{Na}_{32} \mathrm{Cs}_{42}$ presents polyicosahedral packing. The surface shell, formed by $42 \mathrm{Cs}$ atoms, is exactly the same as that of $\mathrm{Cs}_{55}$.

Fig. 4 and found to have the lowest energy, although they contained some structural defects due to kinetic trapping effects during cooling. The structure shown in Fig. 4 was stable upon heating up to the melting point. These observations imply that the simulated annealing method is quite reliable for locating good approximations to the minimum energy structure, and that polyicosahedral ordering is also kinetically favored. In the ground state structure, the core shell is a perfect 45 -atom anti-Mackay $\mathrm{Li}_{13} \mathrm{Na}_{32}$ icosahedron, and is shown in Fig. 1. Each of the $12 \mathrm{Li}$ atoms surrounding the central $\mathrm{Li}$ atom positions itself at the center of a 13-atom icosahedron, built with $6 \mathrm{Li}$ atoms from the inner shell and 6 $\mathrm{Na}$ atoms from the outer shell. The surface shell formed by $\mathrm{Cs}$ atoms grows on top of the $\mathrm{Na}$ shell following again a polyicosahedral packing. Figure 4 shows that the local coordination environment of the two geometrically inequivalent $\mathrm{Na}$ sites is icosahedral, although some of the 13-atom icosahedra are slightly distorted due to the size mismatch. The resulting surface shell of $42 \mathrm{Cs}$ atoms has exactly the same symmetry as the surface shell of $\mathrm{Cs}_{55}$.

Polyicosahedral structures have been independently identified by Rossi et al. ${ }^{21}$ and Rapallo et $a l .{ }^{24}$ for some bimetallic clusters, and by Calvo and Yurtsever ${ }^{28}$ and Doye and Meyer $^{29}$ for some LJ mixtures. In homogeneous clusters, this growing pattern accumulates a very large bond strain which destabilizes it already at quite small sizes, irrespective of the large coordination numbers obtained. On the contrary, in a nanoalloy with a sufficient size mismatch between the components, and with a lower surface tension of the component with larger atomic radius, the atomic species with the shortest equilibrium interatomic distance and/or higher surface tension may form the inner icosahedral core with little or no accumulated strain. Once the strain has been relieved, the large number of nearest-neighbor contacts explains the en- 


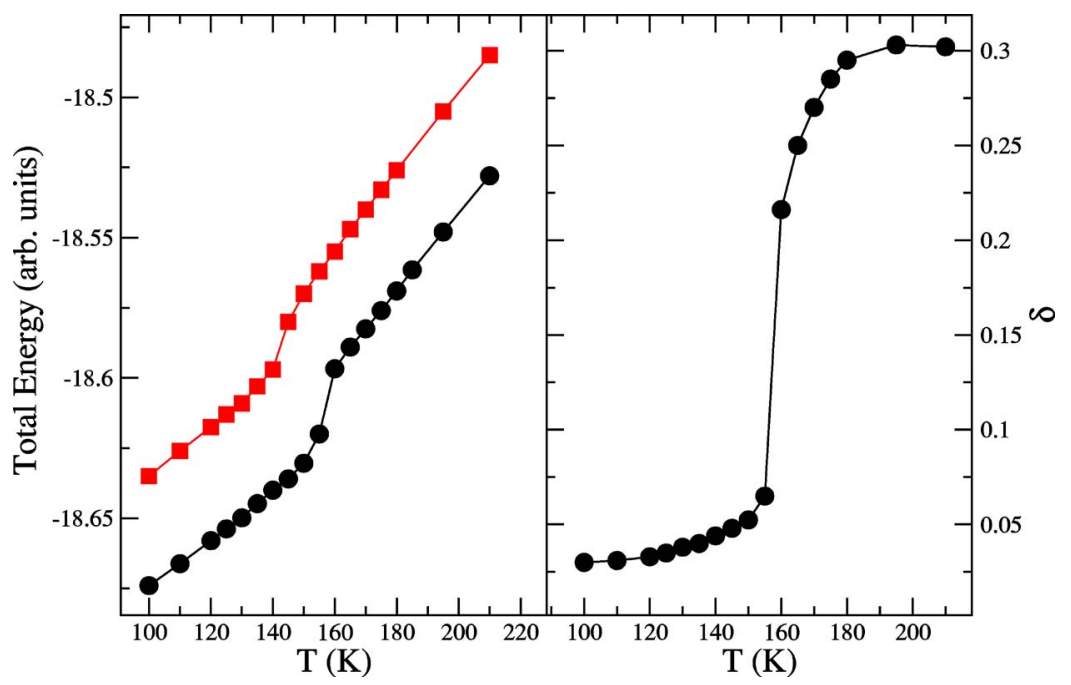

FIG. 5. (Color online) Caloric curve (left) and rms bond-length-fluctuation parameter (right) of $\mathrm{Li}_{13} \mathrm{Na}_{32} \mathrm{Cs}_{42}$ as a function of the cluster internal temperature. The caloric curve of the same cluster, assuming the masses of all atomic species are equal to the Cs atomic mass, is also shown on the left side (square symbols). This curve is displaced along the vertical axes to help visualization.

hanced stability of polyicosahedral structures. In this work, we have shown this mechanism to lead to a perfect core-shell polyicosahedral structure for $\mathrm{Li}_{13} \mathrm{Na}_{32} \mathrm{Cs}_{42}$.

Figure 5 shows the caloric curve and the temperature evolution of $\delta$ for $\mathrm{Li}_{13} \mathrm{Na}_{32} \mathrm{Cs}_{42}$. The cluster melts homogeneously at $160 \mathrm{~K}$. This temperature is much higher than the melting point of $\mathrm{Cs}_{55}$ or the temperatures at which the surfaces of $\mathrm{Na}_{13} \mathrm{Cs}_{42}$ or $\mathrm{Li}_{13} \mathrm{Na}_{30} \mathrm{Cs}_{12}$ melt. ${ }^{35,36}$ Examination of the dynamical trajectories reveals that melting is triggered by the thermal excitation of Cs floater atoms, that is, Cs atoms that move from the surface shell to the next outer layer, generating a hole in the surface shell. The surface disorder associated to the diffusion of the surface hole and the floater atom acts as a catalyst for homogeneous melting, which proceeds in less than $50 \mathrm{ps}$ since the generation of the first floater atom.

We have run nonequilibrium MD simulations in order to generate a thermal gradient along the radial direction, in exactly the same way as we did for $\mathrm{Cs}_{55}$, assigning different local temperatures to the core $\left(\mathrm{Li}_{13} \mathrm{Na}_{32}\right)$ and surface $\left(\mathrm{Cs}_{42}\right)$ shells. The results of these simulations, summarized in Table
I, again demonstrate that the surface shell remains solid upon heating until the core temperature approaches the homogeneous melting temperature of $160 \mathrm{~K}$. With convenient thermal gradients, the surface shell may remain solid even at $192 \mathrm{~K}$, or may melt (induced by the core thermal instability) at only $130 \mathrm{~K}$.

The main reason for the higher thermal stability of the $\mathrm{Cs}_{42}$ surface shell in the ternary mixture is therefore the increased thermal stability of the core shell. However, we notice that, due to the very different masses of $\mathrm{Cs}$ and $\mathrm{Na}$, the atomic vibrations along the radial direction in the solid phase are only weakly coupled. The generation of a floater atom in the Cs surface shell, which is the dynamical mechanism which initiates melting, is largely related to the transfer of collisional momentum across the different radial shells, in analogy with the excitation of capillary waves in an otherwise flat extended surface. In Fig. 6, we show the Fourier transform of the velocity autocorrelation function of each atomic species at several temperatures. In the solid phase, the three different peaks associated to $\mathrm{Li}, \mathrm{Na}$, and $\mathrm{Cs}$ vibrational motions are clearly distinguished, and show only a reduced

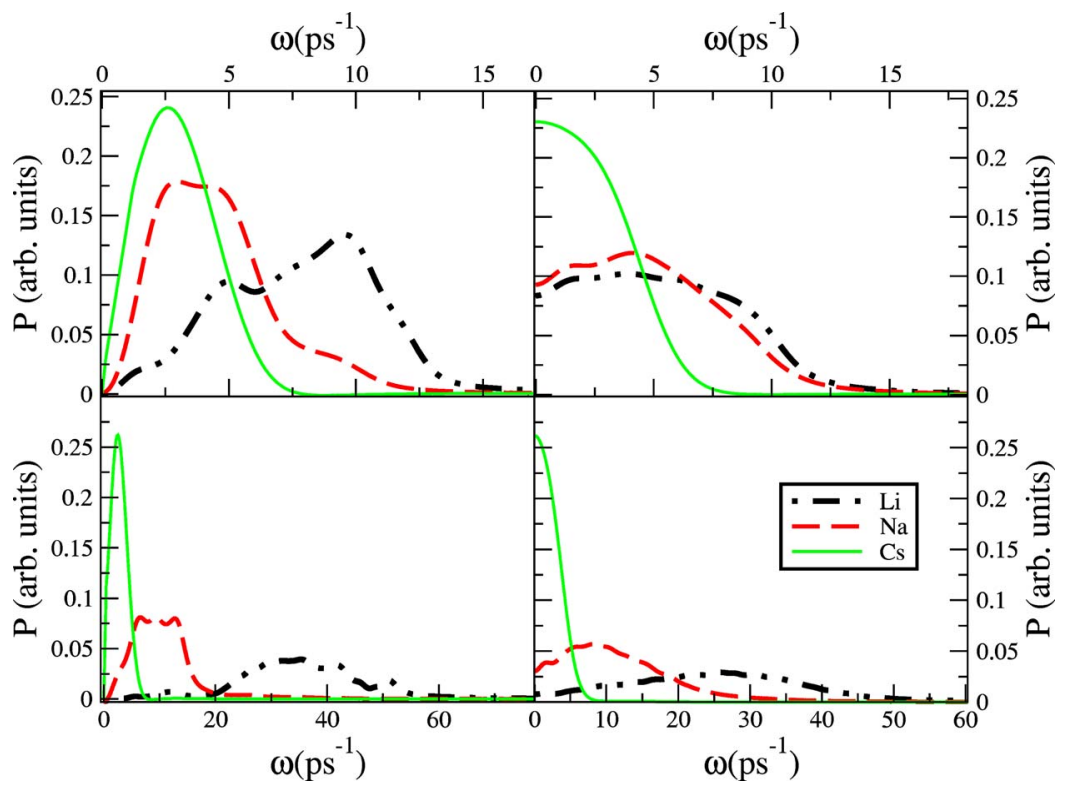

FIG. 6. (Color online) Species-resolved power spectra of $\mathrm{Li}_{13} \mathrm{Na}_{32} \mathrm{Cs}_{42}$ for solid (left side) and liquid (right side) phases. The upper part shows the power spectra obtained when all atomic masses are equal to the $\mathrm{Cs}$ atomic mass, while the lower graphs show the real power spectra. Note the different frequency scales in the two cases. 
amount of overlap. The situation is reminiscent, although to a much lesser degree, of the Born-Oppenheimer approximation for the separate treatment of the electronic and nuclear motions: due to the very large inertia of Cs particles, they cannot efficiently respond to the temporary close approaches of $\mathrm{Na}$ atoms in the radial shell immediatialy below.

To test this idea, we have performed parallel OFAIMD simulations of the melting process for a hypothetical $\mathrm{Li}_{13} \mathrm{Na}_{32} \mathrm{Cs}_{42}$ cluster in which all atomic species have the same mass, equal to the Cs atomic mass (each element retains its electronic features, however, as the pseudopotentials are not changed). Figure 6 shows that the power spectra of the different atomic species are now centered on similar frequencies, which implies a much more efficient radial coupling between atomic vibrations. The caloric curve for this simulation is compared to that of the real $\mathrm{Li}_{13} \mathrm{Na}_{32} \mathrm{Cs}_{42}$ cluster in Fig. 5. Generation of floater atoms at the Cs surface shell starts already at $125 \mathrm{~K}$, and homogeneous melting occurs at $135 \mathrm{~K}$, which is $25 \mathrm{~K}$ lower than the melting point of real $\mathrm{Li}_{13} \mathrm{Na}_{32} \mathrm{Cs}_{42}$. Although the entropy of the solid cluster is considerably higher when the atomic masses are equal (on account of the reduced vibrational frequencies and the easier generation of floater atoms), the entropy of melting, evaluated from Clapeyron's relation, is very similar in both cases because the power spectrum displaces significantly to lower frecuencies also in the liquid state (see Fig. 6). Both homogeneous melting and the thermal excitation of floater atoms occur at a considerably reduced temperature when the masses are equal, due to the much more efficient vibrational coupling between different atomic shells.

Although the total simulation times employed are quite considerable, there is still the chance that the observed sensitivity of the melting transition to the values of the atomic masses is the result of broken ergodicity in our MD simulations. The very weak coupling of radial vibrational modes for the real cluster might just result in very slow kinetics for the melting process, implying that much longer runs would result in melting at lower temperatures. We have not observed any change in the caloric curves by increasing the length of the $155 \mathrm{~K}$ run up to $2 \mathrm{~ns}$. In any case, what our simulation results certainly demonstrate is that the liquid phase is much more easily accessed when the atomic masses are of a comparable magnitude.

Figure 7 shows the radial atomic density distribution at several temperatures. In the solid phase, the radial shells formed by $\mathrm{Li}, \mathrm{Na}$, and $\mathrm{Cs}$ atoms are clearly distinguished. Upon melting, inspection of the atomic trajectories reveals that substantial mixing of $\mathrm{Li}$ and $\mathrm{Na}$ species occurs, and therefore $\mathrm{Li}$ and $\mathrm{Na}$ shells merge into one single core shell. The Cs shell, however, does not mix with the core shell up to the highest temperatures investigated $(240 \mathrm{~K})$, where evaporation of some $\mathrm{Cs}$ atoms is observed. The liquid phase is thus formed by two inmiscible, roughly spherical, liquid layers.

\section{C. $\mathrm{Li}_{55} \mathrm{Cs}_{42}$}

The size mismatch in this cluster is very important, and we soon realized that it is not possible to efficiently pack Cs atoms on top of an icosahedral $\mathrm{Li}_{55}$ core shell following a

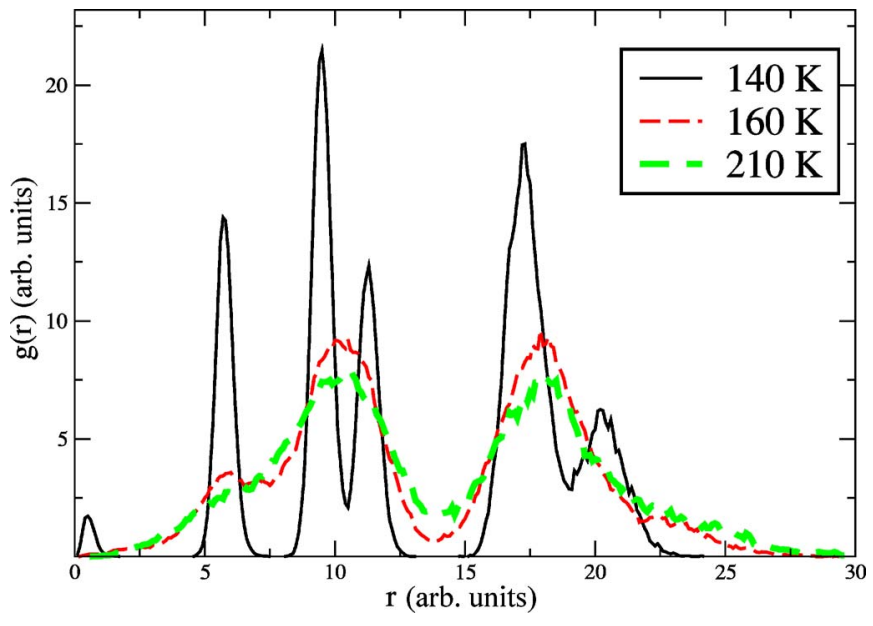

FIG. 7. (Color online) Time averaged radial atomic density distributions of $\mathrm{Li}_{13} \mathrm{Na}_{32} \mathrm{Cs}_{42}$, at some representative temperatures. A deep minimun in $g(r)$ persists in the liquid state, indicating the strong surface seggregation of $\mathrm{Cs}$ atoms. Mixing of $\mathrm{Li}$ and $\mathrm{Na}$ species at the core shell is observed in the liquid phase.

polyicosahedral growing pattern. Therefore we have relied on the results of three simulated annealing runs, starting from a liquid cluster and performed at a cooling rate of $0.1 \mathrm{~K} / \mathrm{ps}$. To better understand the results of these simulated annealing runs, we have also optimized several structures of $\mathrm{Li}_{55}$, in exactly the same way we did above for $\mathrm{Cs}_{55}$.

The lowest energy structure of $\mathrm{Li}_{55}$ is found again to be the compact 2-shell Mackay icosahedron (see Fig. 1). Some other low-energy isomers are shown in Fig. 8, together with the energy differences with respect to the ground state isomer and corresponding results from SIESTA optimizations. Both OFAIMD and SIESTA results predict the same energetic ordering for the different isomers. The first two isomers are based on a decahedral $\mathrm{Li}_{13}$ core shell (shown in the same orientation in Fig. 8 to better appreciate the difference between these isomers), but the surface shell is not perfectly decahedral. In the first isomer, the central ring of $10 \mathrm{Li}$ atoms

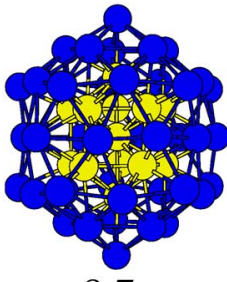

8.7

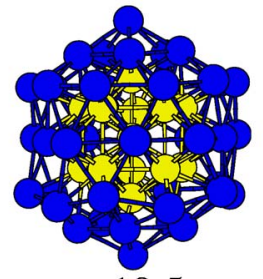

10.5

11.1

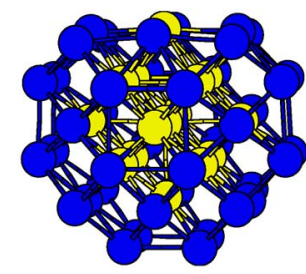

14.1
FIG. 8. (Color online) Low-lying isomers of $\mathrm{Li}_{55}$, together with energy differences, in meV/atom, with respect to the ground state structure (a Mackay icosahedron as shown in Fig. 1). The first (second) row shows OFDFT (SIESTA) results. The first two isomers present a decahedral 13-atom core shell, while core and surface shells are twisted relative to each other so that (100) facets are avoided. These two isomers differ only in the relative orientations of core and surface shells. The isomer on the right side is a fragment of the bcc crystalline lattice. Different colors (shades of gray) are employed for core and surface $\mathrm{Li}$ atoms to help visualization. 

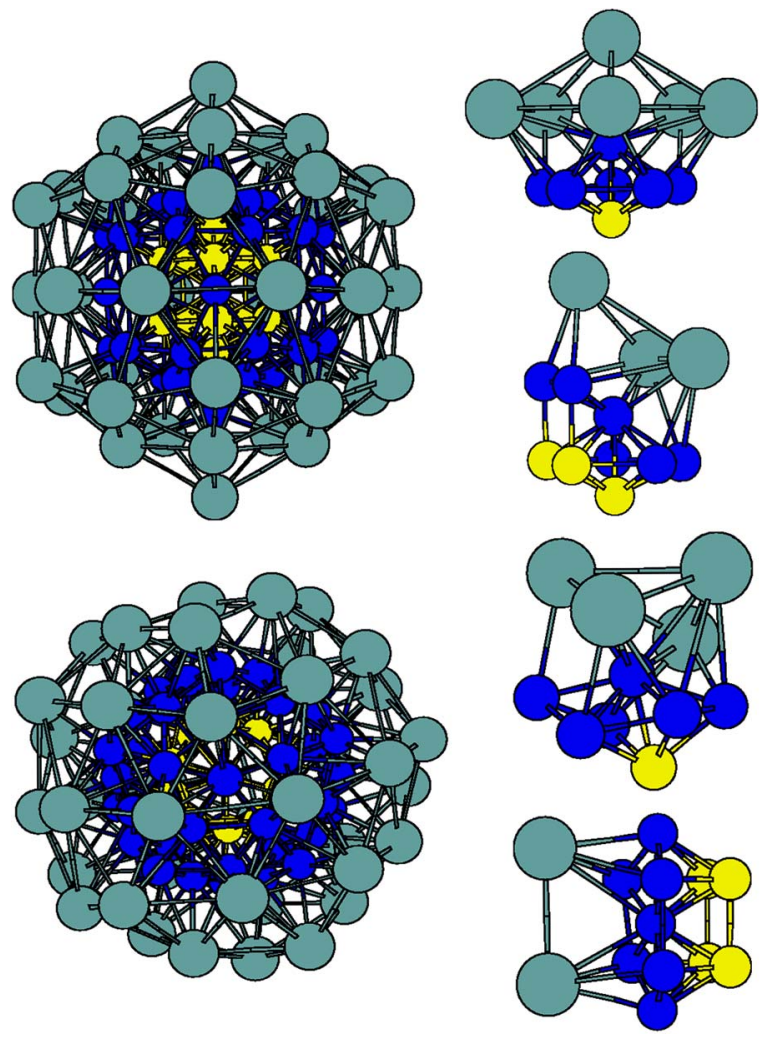

FIG. 9. (Color online) Ground state structure of $\mathrm{Li}_{55} \mathrm{Cs}_{42}$ (upper left), based on a twisted decahedral core as shown in the middle part of Fig. 8. Atoms in different radial shells are given different colors to help visualization. The surface shell, formed by $42 \mathrm{Cs}$ atoms, is close to icosahedral but has some neighboring pentagons due to the twisting distortion. On the right side, we show the different atomic environments of the $\mathrm{Li}$ atoms occupying the outermost radial core shell. Some of them can be viewed as frustrated icosahedra, due to the very large size mismatch in the Li-Cs system. The lower left plot is a low-lying local minimum, based on an icosahedral $\mathrm{Li}_{55}$ core. The orientation of this figure is chosen to easily appreciate the icosahedral symmetry of the core shell. Transformations between these isomers are observed at finite temperatures lower than the melting point (see text).

is slightly rotated (with respect to a perfect 55-atom decahedron) so that all (100) facets are eliminated. In the second isomer, this central 10 -atom ring is exactly in the same orientation (with respect to the 13-atom core) as in a perfect 55-atom decahedron, while the rest of the surface atoms undergo a twisting distortion which again eliminates (100) facets. These isomers are topologically inequivalent due to the different relative orientations of core and surface shells, even though they share the same core and surface shells. Similar twisting distortions have been very recently identified by Noya et $a l .{ }^{52}$ in the structures of cold Na clusters modeled by a Murrell-Mottran potential. They help to lower the cluster energy by exposing only (111) facets. Finally, a fragment of the bcc lattice lies at a higher energy of $14.1 \mathrm{meV} / \mathrm{atom}$.

The three simulated annealing runs for $\mathrm{Li}_{55} \mathrm{Cs}_{42}$ led to the ground state isomer shown in the upper left part of Fig. 9. The core shell is the same decahedral-based $\mathrm{Li}_{55}$ isomer shown in the middle of Fig. 8. We performed two other simu- lated annealing runs, forcing the $\mathrm{Li}_{55}$ core to be either perfectly icosahedral or the isomer shown on the left side of Fig. 8. In these runs, the $\mathrm{Li}$ atoms were assigned a very large mass so that only the Cs shell is liquid at the starting geometry. The best isomer with an icosahedral core is shown in the lower left part of Fig. 9, and is stable upon a reheating/ cooling cycle allowing all atoms to move. The isomer based on the other decahedral isomer is not shown in Fig. 9. It was found to have a higher energy than the ground state isomer shown in Fig. 9 and to spontaneously convert to that ground state isomer upon heating at $100 \mathrm{~K}$. Figure 9 shows that the surface shell formed by $42 \mathrm{Cs}$ atoms is much more symmetrical when grown on top of the decahedral-based $\mathrm{Li}_{55}$ isomer. On the right side, we show the local environments of some geometrically inequivalent $\mathrm{Li}$ atoms forming the surface shell of $\mathrm{Li}_{55}$. Some of those $\mathrm{Li}$ atoms are placed at the center of a 13-atom distorted icosahedron, which shows again a tendency toward polyicosahedral growing. However, due to the very important size mismatch, polyicosahedral ordering is locally frustrated and some Li atoms have a coordination number smaller than 13 . The surface shell is close to but not perfectly icosahedral: one of the five-atom rings around the decahedral symmetry axes is rotated (this rotation is induced by the underlying decahedral symmetry of the core shell) and some neighboring pentagonal rings appear at the surface shell, which are clearly visible in Fig. 9.

Figure 10 shows the results of the melting simulations in the same way as for the previous clusters. The homogeneous melting temperature is approximately $170 \mathrm{~K}$, but both the caloric curve and $\delta$ show some premelting features starting at about $110 \mathrm{~K}$. In order to analyze the origin of this premelting, we have plotted in Fig. 11 the time evolution of shorttime-averages of the atomic equivalence indexes defined in Sec. II. The $\sigma$ indexes provide very detailed structural information. For a solidlike cluster, $\sigma_{i}$ takes a different value for each geometrically inequivalent atom $i$ in the cluster. For example, for a perfect 55-atom icosahedron, the $\sigma_{i}$ values are grouped in four different sets, representing the central atom, the 12 atoms forming the inner icosahedral shell, and the 12 vertex and 30 edge atoms at the cluster surface. On the contrary, the decahedral-based $\mathrm{Li}_{55}$ isomer which forms the core shell of $\mathrm{Li}_{55} \mathrm{Cs}_{42}$ leads to a more complex grouping of $\sigma$-lines, due to a larger number of symmetry inequivalent atomic positions. What Fig. 11 reveals is that the $\mathrm{Li}_{55}$ core shell undergoes back and forth solid-solid transformations between the decahedral-based and icosahedral isomers. A visual examination of the atomic trajectories confirms that these transformations proceed with no appreciable changes in the Cs surface shell (the structure of the $\sigma$-lines corresponding to $\mathrm{Cs}$ atoms changes anyway because the relative orientation of core and surface shells changes, which affects all interatomic distances). The solid-solid transformations proceed via the concerted twisting motion of some rings of atoms about the decahedral symmetry axis, a distortion apparently associated with a low free-energy barrier because it is observed several times during a single MD simulation. An interesting observation is that, once the icosahedral isomer is formed, a new twisting distortion toward the decahedralbased isomer could in principle change the orientation of the decahedral symmetry axis, but this is never observed in our 


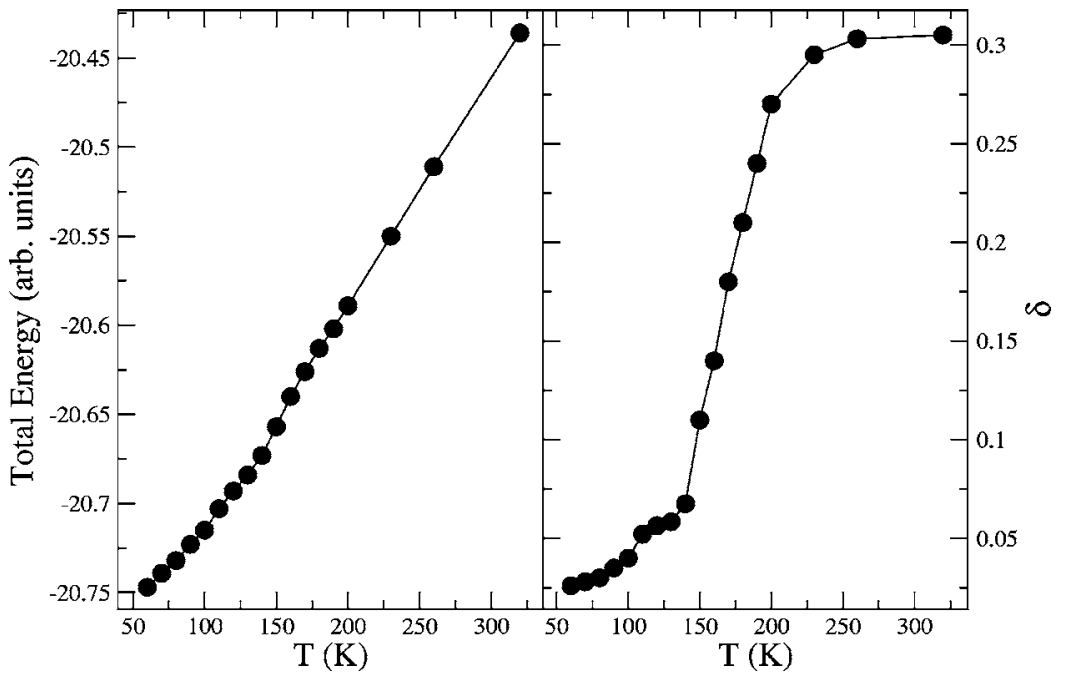

FIG. 10. Caloric curve (left) and rms bondlength-fluctuation parameter (right) of $\mathrm{Li}_{55} \mathrm{Cs}_{42}$, as a function of the cluster internal temperature.

simulations because the Cs surface shell maintains its structure (with a well-defined symmetry axis, because of the twist distortion in the surface shell-see above) during the transformation. In summary, our calculations predict a substantial stabilization of icosahedral isomers similar to that shown in the lower left part of Fig. 9 at finite temperatures. Given the higher energy of the icosahedral isomer, this stabilization must be explained by entropic effects. Temperature-induced transitions from decahedral to icosahedral isomers have been observed for a number of metallic and LJ clusters ${ }^{70}$ because of a reduction in the average vibrational frecuency.

The thermal stabilization of the $\mathrm{Cs}_{42}$ solid surface shell in $\mathrm{Li}_{55} \mathrm{Cs}_{42}$ is even larger than in $\mathrm{Li}_{13} \mathrm{Na}_{32} \mathrm{Cs}_{42}$, studied in the previous section. The liquid phase (which again consists of two inmiscible liquid layers) is stable at $300 \mathrm{~K}$, more than $150 \mathrm{~K}$ above the evaporation threshold of $\mathrm{Cs}_{55}$. Moreover, we again find that a substantial contribution to the melting point increase comes from the mass difference between the constituent atomic species: a melting simulation with all masses set equal to the Cs atomic mass resulted in homogeneous melting at a temperature $30 \mathrm{~K}$ lower than the real melting point. Therefore this seems to be a rather general phenomenon, applicable to other core-shell nanoparticles, not necessarily formed by alkali metals. The melting transition is again driven by the thermal instability of the core shell $\left(\mathrm{Li}_{55}\right)$ rather than the surface shell $\left(\mathrm{Cs}_{42}\right)$, as demonstrated by the nonequilibrium MD results shown in Table I. This also seems to be a rather general phenomenon in alkali clusters when the surface shell is complete, namely, when it does not contain any structural defects. The entropy of melting is much lower than that of $\mathrm{Li}_{13} \mathrm{Na}_{32} \mathrm{Cs}_{42}$. In the ternary cluster, mixing of $\mathrm{Li}$ and $\mathrm{Na}$ species upon melting is a source of entropy increase not present in $\mathrm{Li}_{55} \mathrm{Cs}_{42}$. Moreover, the entropy of $\mathrm{Li}_{55} \mathrm{Cs}_{42}$ increases considerably before melting due to the solid-solid isomerizations in the core shell.

\section{SUMMARY AND DISCUSSION}

In this paper, orbital-free DFT molecular dynamics simulations have been employed in order to analyze the structure and the mechanisms of melting in two alkali nanoalloys, namely $\mathrm{Li}_{13} \mathrm{Na}_{32} \mathrm{Cs}_{42}$ and $\mathrm{Li}_{55} \mathrm{Cs}_{42}$, and in a homogeneous alkali cluster, $\mathrm{Cs}_{55}$. The results from the structural analysis show that $\mathrm{Cs}_{55}$ is a compact 2-shell Mackay icosahedron, as

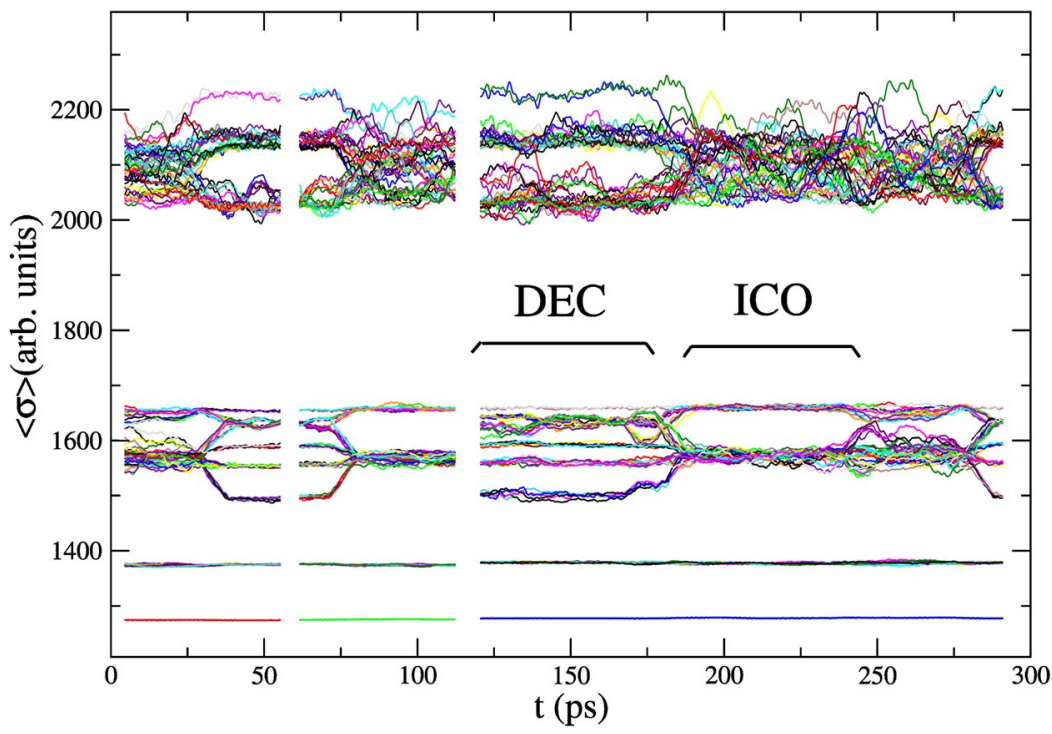

FIG. 11. (Color online) Time evolution of atomic equivalence indexes of $\mathrm{Li}_{55} \mathrm{Cs}_{42}$, averaged over 3000 time steps ( 9 ps), showing the back and forth solid-solid transformations prior to the melting transition. Portions from three different MD runs in the temperature range 110-130 K are shown together. The legends "ICO" and "DEC" help to differenciate between the sets of $\sigma$ lines corresponding to icosahedral and ground state isomer, respectively. 
expected from a simple alkali metal. $\mathrm{Li}_{13} \mathrm{Na}_{32} \mathrm{Cs}_{42}$ adopts a compact structure based on polyicosahedral growing, with complete surface seggregation of Cs atoms. The core shell $\left(\mathrm{Li}_{13} \mathrm{Na}_{32}\right)$ is a perfect 45 -atom anti-Mackay icosahedron, and the surface shell $\left(\mathrm{Cs}_{42}\right)$ forms an anti-Mackay overlayer on top of the Na shell. The structure of the $\mathrm{Cs}_{42}$ shell thus formed is exactly the same as that of the surface shell of $\mathrm{Cs}_{55}$. The preference for polyicosahedral growing has been rationalized in terms of surface tension and size mismatch arguments. For $\mathrm{Li}_{55} \mathrm{Cs}_{42}$, the size mismatch is too large and polyicosahedral ordering is frustrated. In the ground state structure, the core shell $\left(\mathrm{Li}_{55}\right)$ has a mixture of decahedral and icosahedral symmetries, and the surface shell (again $\mathrm{Cs}_{42}$ ) grows on top of the core shell with a clear tendency towards polyicosahedral ordering. The structure of this surface shell is almost the same (except by a twist distortion) as the surface shell of $\mathrm{Cs}_{55}$. The common feature in these structures is therefore the surface shell, which is in all cases formed by $42 \mathrm{Cs}$ atoms, has monatomic thickness (there are no floater atoms outside the surface shell), and is perfectly closed (there are no vacant atomic sites). This $\mathrm{Cs}_{42}$ surface shell encapsulates different core shells $\left(\mathrm{Cs}_{13}, \mathrm{Li}_{13} \mathrm{Na}_{32}, \mathrm{Li}_{55}\right)$.

The analysis of the melting transition in these clusters reveals that the thermal stability of the $\mathrm{Cs}_{42}$ surface shell is much higher in the two nanoalloys than in $\mathrm{Cs}_{55}$. Nevertheless, we have found some systematics in the melting behavior of the three clusters: (1) they melt homogeneously, that is, without a separate surface melting stage; and (2) the melting transition starts at the surface, and proceeds through the generation of floater Cs atoms, that is, Cs atoms that move from the surface shell to the next outer layer. Diffusion of these floater atoms and also of the holes left in the surface layer lead to homogeneous melting in approximately $50 \mathrm{ps}$. The absence of a separate surface melting stage is suggestive of a high thermal stability for the $\mathrm{Cs}_{42}$ surface shell.

In order to test this idea, we have exploited the freedom offered by computer experiments and run MD simulations on thermodynamic conditions which are either not realizable or not easily controlled in real experiments. In a first set of computer experiments, nonequilibrium MD simulations have been performed by scaling the velocities of core and surface atoms by different factors. In this way, a thermal gradient is generated along the radial cluster direction and different local temperatures may be assigned to core and surface shells. In all cases, we have found that the clusters melt when the core temperature approaches the homogeneous melting temperature $T_{m}$, irrespective of the value of the surface temperature. The surface shell temperature may be significantly higher than $T_{m}$ at the melting point, which implies a higher thermal stability for the surface shell, as compared to the core shell. $T_{\text {surface }}$ may be also significantly lower than $T_{m}$ at the melting point, implying that melting of the surface shell is driven by the thermal instability of the solid core shell. Thus what is common in the three clusters is that the surface shell remains stable upon heating until the core shell is ther- mally unstable. Even if melting nucleates at the surface shell, as explained above, it is the thermal instability of the core shell which ultimately triggers the melting transition.

The different melting points of $\mathrm{Cs}_{55}, \mathrm{Li}_{13} \mathrm{Na}_{32} \mathrm{Cs}_{42}$, and $\mathrm{Li}_{55} \mathrm{Cs}_{42}$ is thus mainly a consequence of the different thermal stabilities of the core shells, which are different in the three clusters. We have also found that a substantial contribution to the higher thermal stability of the nanoalloys (as compared to $\mathrm{Cs}_{55}$ ) comes from that large mass difference between the atomic species. Generation of floater atoms at the Cs surface shell, which is the precursor of homogeneous melting, depends significantly on the degree of coupling between the atomic vibrations of different radial atomic shells. The vibrational densities of states (VDOS) of nanoalloys reveal that there is very little coupling between the surface (Cs) and core $(\mathrm{Li}, \mathrm{Na})$ vibrational modes. The very large inertia of Cs atoms makes the energy/momentum transfer between radial atomic shells very inefficient. To explicitly demonstrate this, we have run a set of MD heating simulations in which the masses of all atomic species are set equal to the Cs atomic mass (leaving the electronic degrees of freedom unchanged). The VDOS of the different atomic species in the solid-phase nanoalloys are then centered on very similar frequencies, and the coupling of vibrational modes is thus much more efficient. The melting point in these runs is considerably lower (up to $30 \mathrm{~K}$ lower for $\mathrm{Li}_{55} \mathrm{Cs}_{42}$ ) than the real melting point because the excitation of floater atoms at the surface shell occurs at much reduced temperatures.

To summarize, there are several reasons for the high thermal stability of the surface shell in $\mathrm{Li}_{13} \mathrm{Na}_{32} \mathrm{Cs}_{42}$ and $\mathrm{Li}_{55} \mathrm{Cs}_{42}$, as compared to $\mathrm{Cs}_{55}$. First of all, the strong preference for surface seggregation of Cs atoms (which is maintained even in the liquid state) efficiently closes one possible diffusion channel, namely, diffusion of Cs atoms towards the inner cluster shells. In the second place, surface diffusion is largely impeded because the surface shell is of monatomic thickness and complete, that is, there are no vacant sites which could enhance surface diffusion. The only diffusion channel which is accesible to the solid surface shell is thus the thermal generation of intrinsic defects (floater atoms with associated surface shell atomic vacancies), and activation of this mechanism is also significantly impeded by poor momentum transfer between radial shells. Finally, in all three clusters, generation of floater surface atoms occurs when the average kinetic energy per atom in the core shell is that corresponding to the homogeneous melting temperature, which explains the absence of a separate surface melting stage in the melting process.

\section{ACKNOWLEDGMENTS}

This work was supported by DGES (Project MAT200204393-C02-01). A.A. also acknowledges financial support from the Spanish Ministry of Science and Technology under the Ramón y Cajal program. 
${ }^{1}$ A. M. Molenbroek and J. K. Norskov, J. Phys. Chem. B 105, 5450 (2001).

${ }^{2}$ H. Yasuda and H. Mori, Phys. Rev. Lett. 69, 3747 (1992).

${ }^{3}$ H. Portales, L. Saviot, E. Duval, M. Gaudry, E. Cottancin, M. Pellarin, J. Lermé, and M. Broyer, Phys. Rev. B 65, 165422 (2002).

${ }^{4}$ M. Moskovits, I. Srnova-Sloufova, and B. Vickova, J. Chem. Phys. 116, 10435 (2002).

${ }^{5}$ H. Tada, F. Suzuki, S. Ito, T. Akita, K. Tanaka, T. Kawahara, and H. Kobayashi, J. Phys. Chem. B 106, 8714 (2002).

${ }^{6}$ J. P. Wilcoxon and P. P. Provencio, J. Am. Chem. Soc. 126, 6402 (2004).

${ }^{7}$ J. L. Rodríguez-López, J. M. Montejano-Carrizales, U. Pal, J. F. Sánchez-Ramírez, H. E. Troiani, D. García, M. Miki-Yoshida, and M. José-Yacamán, Phys. Rev. Lett. 92, 196102 (2004).

${ }^{8}$ J. G. Lee and H. Mori, Phys. Rev. B 70, 144105 (2004).

${ }^{9}$ M. J. López, P. A. Marcos, and J. A. Alonso, J. Chem. Phys. 104, 1056 (1996).

${ }^{10}$ J. Jellinek and E. B. Krissinel, Chem. Phys. Lett. 258, 283 (1996); E. B. Krissinel and J. Jellinek, ibid. 272, 301 (1997).

${ }^{11}$ Z. H. Jin, H. W. Sheng, and K. Lu, Phys. Rev. B 60, 141 (1999).

${ }^{12}$ D. S. Mainardi and P. P. Balbuena, Int. J. Quantum Chem. 85, 580 (2001)

${ }^{13}$ S. Huang and P. P. Balbuena, J. Phys. Chem. B 106, 7225 (2002).

${ }^{14}$ S. Darby, T. V. Mortimer-Jones, R. L. Johnston, and C. Roberts, J. Chem. Phys. 116, 1536 (2002).

${ }^{15}$ N. T. Wilson and R. L. Johnston, J. Mater. Chem. 12, 2913 (2002).

${ }^{16}$ Y. G. Chushak and L. S. Bartell, J. Phys. Chem. B 107, 3747 (2003).

${ }^{17}$ K. Joshi and D. G. Kanhere, J. Chem. Phys. 119, 12301 (2003).

${ }^{18}$ M. S. Bailey, N. T. Wilson, C. Roberts, and R. L. Johnston, Eur. Phys. J. B 25, 41 (2003).

${ }^{19}$ F. Baletto, C. Mottet, and R. Ferrando, Phys. Rev. B 66, 155420 (2002); Phys. Rev. Lett. 90, 135504 (2003).

${ }^{20}$ F. Baletto, C. Mottet, A. Rapallo, G. Rossi, and R. Ferrando, Surf. Sci. 566-568, 192 (2004).

${ }^{21}$ G. Rossi, A. Rapallo, C. Mottet, A. Fortunelli, F. Baletto, and R. Ferrando, Phys. Rev. Lett. 93, 105503 (2004).

${ }^{22}$ S. K. R. S. Sankaranarayanan, V. R. Bhethanabotla, and B. Joseph, Phys. Rev. B 71, 195415 (2005).

${ }^{23}$ M. Polak and L. Rubinovich, Phys. Rev. B 71, 125426 (2005).

${ }^{24}$ A. Rapallo, G. Rossi, R. Ferrando, A. Fortunelli, B. C. Curley, L. D. Lloyd, G. M. Tarbuck, and R. L. Johnston, J. Chem. Phys. 122, 194308 (2005).

${ }^{25}$ G. Rossi, R. Ferrando, A. Rapallo, A. Fortunelli, B. C. Curley, L. D. Lloyd, and R. L. Johnston, J. Chem. Phys. 122, 194309 (2005).

${ }^{26}$ R. Ferrando, A. Fortunelli, and G. Rossi, Phys. Rev. B 72, 085449 (2005)

${ }^{27}$ C. Mottet, G. Rossi, F. Baletto, and R. Ferrando, Phys. Rev. Lett. 95, 035501 (2005).

${ }^{28}$ F. Calvo and E. Yurtsever, Phys. Rev. B 70, 045423 (2004).

${ }^{29}$ J. P. K. Doye and L. Meyer, Phys. Rev. Lett. 95, 063401 (2005).

${ }^{30}$ M. D. Deshpande, D. G. Kanhere, P. V. Panat, I. Vasiliev, and R. M. Martin, Phys. Rev. A 65, 053204 (2002); M. D. Deshpande, D. G. Kanhere, I. Vasiliev, and R. M. Martin, ibid. 65, 033202 (2002).

${ }^{31}$ K. Joshi and D. G. Kanhere, Phys. Rev. A 65, 043203 (2002).

${ }^{32}$ S. Chacko, D. G. Kanhere, and V. V. Paranjape, Phys. Rev. A 70,
023204 (2004).

${ }^{33}$ A. Aguado, L. E. González, and J. M. López, J. Phys. Chem. B 108, 11722 (2004); A. Aguado, S. Núñez and J. M. López, Comput. Mater. Sci. (to be published).

${ }^{34}$ A. Aguado, D. J. González, L. E. González, and J. M. López, in Progress in Chemical Physics Research (Nova Science Publishers, New York, in press).

${ }^{35}$ A. Aguado and J. M. López, Phys. Rev. B 71, 075415 (2005).

${ }^{36}$ A. Aguado and J. M. López, J. Chem. Theory Comput. 1, 299 (2005).

${ }^{37}$ M. Schmidt, R. Kusche, W. Kronmüller, B. von Issendorff, and H. Haberland, Phys. Rev. Lett. 79, 99 (1997); M. Schmidt, R. Kusche, B. von Issendorff, and H. Haberland, Nature (London) 393, 238 (1998); R. Kusche, Th. Hippler, M. Schmidt, B. von Issendorff, and H. Haberland, Eur. Phys. J. D 9, 1 (1999).

${ }^{38}$ M. Schmidt and H. Haberland, C. R. Phys. 3, 327 (2002); M. Schmidt, J. Donges, Th. Hippler, and H. Haberland, Phys. Rev. Lett. 90, 103401 (2003).

${ }^{39}$ H. Haberland, Th. Hippler, J. Donges, O. Kostko, M. Schmidt, and B. von Issendorff, Phys. Rev. Lett. 94, 035701 (2005).

${ }^{40}$ A. Aguado, J. M. López, J. A. Alonso, and M. J. Stott, J. Chem. Phys. 111, 6026 (1999).

${ }^{41}$ A. Aguado, J. M. López, J. A. Alonso, and M. J. Stott, J. Phys. Chem. B 105, 2386 (2001).

${ }^{42}$ A. Aguado, L. M. Molina, J. M. López, and J. A. Alonso, Eur. Phys. J. D 15, 221 (2001).

${ }^{43}$ A. Aguado, Phys. Rev. B 63, 115404 (2001).

${ }^{44}$ A. Rytkönen, H. Häkkinen, and M. Manninen, Phys. Rev. Lett. 80, 3940 (1998).

${ }^{45}$ F. Calvo and F. Spiegelmann, Phys. Rev. Lett. 82, 2270 (1999); J. Chem. Phys. 112, 2888 (2000).

${ }^{46}$ F. Calvo and F. Spiegelmann, J. Chem. Phys. 120, 9684 (2004).

${ }^{47}$ K. Manninen, A. Rytkönen, and M. Manninen, Eur. Phys. J. D 29, 39 (2004).

${ }^{48}$ K. Manninen, H. Häkkinen, and M. Manninen, Phys. Rev. A 70, 023203 (2004).

${ }^{49}$ S. Chacko, D. G. Kanhere, and S. A. Blundell, Phys. Rev. B 71, 155407 (2005).

${ }^{50}$ A. Aguado and J. M. López, Phys. Rev. Lett. 94, 233401 (2005).

${ }^{51}$ A. Aguado, J. Phys. Chem. B 109, 13043 (2005).

${ }^{52}$ E. G. Noya, J. P. K. Doye, and D. J. Wales, cond-mat/0506329 (unpublished).

${ }^{53}$ P. Hohenberg and W. Kohn, Phys. Rev. 136, B864 (1964).

${ }^{54}$ W. Kohn and L. J. Sham, Phys. Rev. 140, A1133 (1965).

${ }^{55}$ Theory of the Inhomogeneous Electron Gas, edited by S. Lundqvist and N. H. March (Plenum Press, New York, 1983).

${ }^{56}$ W. Yang, Phys. Rev. A 34, 4575 (1986).

${ }^{57}$ J. P. Perdew, Phys. Lett. A 165, 79 (1992).

${ }^{58}$ J. P. Perdew and A. Zunger, Phys. Rev. B 23, 5048 (1981).

${ }^{59}$ D. M. Ceperley and B. J. Alder, Phys. Rev. Lett. 45, 566 (1980).

${ }^{60}$ C. Fiolhais, J. P. Perdew, S. Q. Armster, J. M. MacLaren, and M. Brajczewska, Phys. Rev. B 51, 14001 (1995); 53, 13193 (1996).

${ }^{61}$ J. M. Soler, E. Artacho, J. D. Gale, A. García, J. Junquera, P. Ordejón, and D. Sánchez-Portal, J. Phys.: Condens. Matter 14, 2475 (2002).

${ }^{62}$ B. Zhou and E. A. Carter, J. Chem. Phys. 122, 184108 (2005).

${ }^{63}$ R. Car, and M. Parrinello, Phys. Rev. Lett. 55, 2471 (1985); M. C. Payne, M. P. Teter, D. C. Allan, T. A. Arias, and J. D. Joannopoulos, Rev. Mod. Phys. 64, 1045 (1992).

${ }^{64}$ L. Verlet, Phys. Rev. 159, 98 (1967); W. C. Swope and H. C. 
Andersen, J. Chem. Phys. 76, 637 (1982).

${ }^{65}$ G. P. Morriss and C. P. Dettmann, Chaos 8, 321 (1998).

${ }^{66}$ P. Minary, G. J. Martyna, and M. E. Tuckerman, J. Chem. Phys. 118, 2510 (2003).

${ }^{67}$ M. D'Alessandro, A. Tenenbaum, and A. Amadei, J. Phys. Chem. B 106, 5050 (2002).

${ }^{68}$ A. Amadei, G. Chillemi, M. A. Ceruso, A. Grottesi, and A. Di
Nola, J. Chem. Phys. 112, 9 (2000).

${ }^{69}$ V. Bonacić-Koutecký, J. Jellinek, M. Wiechert, and P. Fantucci, J. Chem. Phys. 107, 6321 (1997); D. Reichardt, V. BonacićKoutecký, P. Fantucci, and J. Jellinek, Chem. Phys. Lett. 279, 129 (1997).

${ }^{70}$ F. Baletto and R. Ferrando, Rev. Mod. Phys. 77, 371 (2005). 